II.

\title{
Ueber Eisenbahstatistik
}

aus Anlass der Berner Session der internationalen fachmiinnischen Kommission im September 1878.

Nach einem Yortrage in der etatistisch-volkswirthschaftlichen Gesellschaft in Basel.

Von

W. Heusler-VonderMühll,

Mitglied des Direktoriuuns der schweizerischen Centralbahn ').

Die internationale fachmännische Kommission für Eisenbahnstatistik ist von dem Allgemeinen statistischen Kongress bei seiner $\mathrm{Zu}$ sammenkunft in Budapest im Jahre 1876 eingesetzt worden, um die Frage ' zu' untersuchen, auf welchen Grundlagen eine internationale Eiseubabnstatistik aufzustellen sei, nachdem man sich an mehreren früheren Versammlungen mit diesem Gegenstande beschäftigt hatte, aber nur zu dem negativen Resultate gelangt war, dass die bestebenden einzelnen oder nationalen Statistiken für den angestrebten Zweck nicht hinreichend seien.

Wenn vielleicht in frühereu Jalıren die Frage nur ein mehr wissenschaftliches Interesse batte, so erlielt sie dagegen in den letzten Jahren in un so böherem Maasse eine allgemeine und praktische Beleutung, je mehr der vielenorts eintretende Nothstand der Eisenbahnen cine genaue Untersuchung ihrer Verhältnisse auf die Tagesordnung braclite, und je mehr damit in Zusammenhang die Frage „Staatsbahnen oder Privatbahnen“ zur Erörterung gelangte. Denn es liegt auf der Ilaud, dass diese Frage, die den Kern aller Eisenbahndebatten und Polemiken bildet, nicht ausschliesslich vom politischen Standpunkte aus oder nur an der Hand allgemeiner Theorien beurtheilt werden darf, soudern dass dabei die reellen Verhältnisse, die Resultate, welche bis jetzt mit dem Staatsbau und Betrieb und mit

1) Der Verfasser hat s. Z. an den Verhandlungen in Bern ais Vertroter der 8.C.B. Theil genommen.

XXXIV. 
dem Bau und Betrieb durch Privatgesellschaften erreicht worden sind, wohl ebeu so stark in Betracht kommen miissen. Diese thatsiichlichen Resultate sollte nun in erster Linie die Statistik zu liefern im Stande scin, und es war demnach ein ganz natürlicher Gedanke, nachdem maI sich uberzeugt hatte, dass dics nicht der Fall sei, zu versuchen, ob nicht diese Lücke durch das Zusammenwirken von Fachmï̈neru aller Nationen ausgefüllt werden köunte.

Wie alle Statistiken, so hat auch die Eisenbahnstatistik zwei Aufgaben zu erfüllen:

die eine ist die Vergleichung der Resultate einer und derselben Balın zu verschiedenen Zeiten;

die andere ist die Vergleichung der Resultate verschiedener Bahnen für einen und denselben Zeitraum.

Fast ausnahmslos tritt der erste Zweck in den Vordergrund, wenn cine einzelue Bahnverwaltung die Resultate ihres Betriebes statistisch orduet, und es ist eiuleuchtend, dass dieser Zweck um so besser erreicht wird, je weniger an den Formulareu und Tabellen im Laufe der Jahre geündert wird, weil dadurch die gegebenen Zahlen immer dieselbe Bedeutung beibehalten; und es kommt auch in der That weniger darauf an, dass die absolut richtigsten Einheiten zur Vergleichung genommen werden, als darauf, dass dieselben fïr einen möglichst langen Zeitraum immer die nämlichen bleiben. Die einzelıen Verwaltungen werden also - beiläufig gesagt - der Natur der Sache nach allen Abänderungen in der Statistik, welche immer auch eine solche in ihrem Rechnungswesen nach sich ziehen, von vornherein eher abgeneigt sein.

Viel weiter gehenden Anforderungen haben aber die Statistiken der einzelnen Gesellschaften zu entsprechen, wenn deren Ziffern auch unter sich vergleichbar sein sollen; denn dann muss die Bedeutung dieser letztern für alle Bahnen absolut die nämliche sein, wenn ein richtiges Resultat erlangt werden soll. Um diesen zweiten Zweck bis zu einem gewissen Grade wenigstens zu erreichen, haben sich denn auch in verschiedenen Verkehrsgebieten theils freiwillig, theils auf Anregung der Regierungen hin, die Bahnen auf eine einheitliche, nationale Statistik vereinigt, und so besteben die Preussische Statistik, diejenige des Vereins deutscher Eisenbahnverwaltungen, die Schweizerische, die Französische, die Englische Statistik, welche alle Angaben liefern, die je für das betreffende Gebiet unter einander verglichen werden können, welche 
dagegen in ihrer Anlage von einem Lande zum andern ganz ausserordentlich abweichen.

Das Bestehen dieser nationalen Statistiken ist aber elher ein Hinderniss für die Erstellung einer internationalen Statistik, weil einerseits jeder Staat das für ilın Passendste und Zweckdionlichste erreicht zu haben glaubt und also nicht gerne das Errungene preisgibt, und weil anderseits die zwischen den verschicdenen lormularen bestehenden Differenzen grossentheils in den Verwaltungs- und Betriebsgrundsützen, in der Organisation und: der Gesetzgebung der einzelnen Staaten ihre Begründung haben und desshalb auch sehr schwer zu vermitteln sind.

Die Aufgabe der Kommission war also eine sehr schwierige, uud wir werden auch sehon, dass die Lösung derselben trotz möglichsten Entgegenkommens der anwesenden Mitglieder noch in weitem Felde liegt.

Bevor wir auf die Arbeiten der internationalen Kommission eingehen, erscheint es angezeigt, einige Bemerkungen vorauszuschicken uber die Eisenbahustatistik im Allgemeinen und über die Schwierigkciten, die die Aufstellung wie der Gebrauch derselben bietet.

Vor Allem ist es der Unstand, dass die Einheiten, nach welchen gemessen wird und nothgedrungen genessen werden muss, ihrer Natur nach nicht überall den nümlicheu Werth haben. Es ist hier selbstverständlich nicht von der Verschiedenheit von Maass, Gewicht und Geld in den verschiedenen Länderu Rede, da die Reduktion eine einfache Rechnungsoperation ist, obwohl bei der gegenwärtig vielenorts herrschenden Papierwirthschaft auch die Umrechunngskurse der Rubel, der Gulden und Liren etc. zu Unsicherheit Anlass geben könneu, sondern was gemeint ist, das sind die oft ganz enormen Differenzen an inuerem Werthe, an Leistungsfähigkeit von gleich benanuten Einlıeiten in den verschiedeuen Ländern. Nehmen wir z. B. einen Kilometer Bahn in Englaud, und einen solchen in Oesterreich, so können dieselben nicht ohne Weiteres mit einander verglichen werden nit Bezug auf Baukosten oder auf das Personal, das durchschnittlich zum Betrieb erfordert wird, sondern es sollte jeweilen auch gegenubergestellt werden dic Ausrüstung der Bahnen an Geleisen und Rollmaterial und die durchschnittliche Leistung an abgelassenen Zügen u. s. w.

Der englische Kilometer hat allerdings in Durchschnitt Fr. 700,000') an Baukosten erfordert, und der österrcichische kaum die Hülfte $\left(\right.$ Fr. 340,000) ${ }^{1}$ ); dafür sind aber auch in England die Bahnen zu mehr

1) Ia uigi Bodio, Appunti di Statistica Ferroviaria pag. 9. 
a]s $50 \%$ zweigeleisig und besitzen per Myriameter (10 Kil.) 5 Lokomotiven und 150 Wagen und werden durchschnittlich Tag für Tag von 34 Zügen befahren, während von den österreichischen Bahnen nur $10 \%$ zweigeleisig sind, auf 10 Kilometer nur 2 Lokomotiveu und 50 Wagen entfallen und eudlich per Tag kaum 12 Zuge uber das ganze Netz gehen ${ }^{1}$ ).

Oder nehmen wir die Personentaxen per Kilometer, so sind sie in England iın Durchschnitt um vielleicht $50 \%$ höher als in Oesterreich, wo sie wie in der Schweiz ca. 5-51/2 cs. betragen; un aber ein richtiges Urtheil zu füllen, muss berücksichtigt werden, dass in England die Falırgeschwindigkeit viel grösser ist und auch viel mehr Roisende in der I. und II. Klasse reisen als in Oesterreich, wo z. B. die I. Klasse nur von ca. $3 \%$ aller Reisenden benutzt wird. Oder wenn man die Baukosten der Jurabahn und der S.C.B. mit einander vergleicht, welche ca Fr. 260,000 resp. Fr. 360,000 per Kilometer betragen, so sollte man nicht ausser Augen lassen, dass die schweizer. Centralbahn zu ca. 1/4 ihrer Lünge zweispurig ist, die Jurabahn dagegen fast durchweg einspurig, und hauptsächlich, dass alle grossen Bahuhöfo des S.C.B.-Netzes von der S.C.B. selbst gebaut und bezahlt sind (mit Ausnahme von Biel), während die Jurabahn in Basel, Bern, Luzern, Neuenburg nur zur Miethe ist, das entsprechende, sehr bedeutende Baukapital also hat sparen können.

Gauz analoge Verschiedenheiten ergeben sich, wenn die Betriebskosten per Zugskilometer verglichen werden; es kommt dabei darauf an, ob die Züge grundsätzlich lang gemacht werden, dagegen wenig zahlreich sind, wie in Deutschland und Frankreich, oder ob, wie in England, auf schnelle Beförderung (von Gütern wie Personen) ein Hauptgewicht gelegt wird, und desshalb in kurzen Zwischenräumen viele kleinere Züge auf eimander folgen.

Zu dieser ersten Schwierigkeit, dass die Einheitenn, nach denen gemessen werden muss, ihrer Natur nach in den verschiedenen Ländern (ubrigens auch oft, doch in geringercm Grade, von einer Bahn des nämlichen Landes zur andern) nicht denselben Werth haben, einer Schwierigkeit, die unter gar keinen Unständen beseitigt werden kann, tritt die zweite hinzu, die allerdings nicht ebenso absolut unabweisbar ist, nämlich die verschiedenartige Berechnungsweise vieler Zahlen der Statistik.

Diese Verschiedenheit tritt hauptsächlich zu Tage bei dem finan-

1) Luigi Bodi o, Appunti di Statistica Ferroviaria pag. 22, 23. 
zicllen Thcile der Statistik, bei den Angaben aber Einnahmen und Ausgaben und das Verhältniss der letztern zu den erstern.

Ein beliebter Maassstab für die Vorzüglichkeit einer Verwaltung ist das Verhältniss der Ausgaben zu den Einnahmen, und man geht sogar so weit, gewisse Prozentsätze - z. B. 50\%, als eine Art Norm aufzustellen, über welche die Ausgaben nicht hinausgehen sollen. Ganz abgeselien davon, dass dieser Prozentsatz ebensovicl von den Einnalımen, auf welche die Verwaltung viel weniger Einfluss hat, abhängt als von den Ausgaben, und dass bei hohen Taxen die nämlicheu Betriebskosten einen niedrigeren Prozentsatz der Einnahmen ausmachen als bei niedern Taxen, - wenn die cine Bahn für einen 'Transport Fr. 100 erhält, eine andere Bahn dagegen nur Fr. 80 und bei beiden Bahnen der Transport Fr. 40 kostet, so machen die Ausgaben bei der ersten $40 \%$, bei der andern $50 \%$ der Einuahmen aus, obgleich beide faktisch ebenso billig betreiben - ganz abgesehen von diesem Umstande, der mehr in die Kategorie der unabweisbaren Verschiedenheiten gehört, und allein schon genügt, um dem Prozentverhailtuiss der Ausgaben zu den Einnahmen die Geltung als absoluter Maassstab zu benehmen, werden aber auch unter Einnahmen und Ausgaben fast nirgends genau dieselben Posten verstanden, und man kann, wenn man will, mit einiger Geschicklichkeit diesen Prozentsatz in der Rechnung immer auf einem anständig ticfen Niveau halten.

Im Grunde genommen, sollten der Berechnung desselben nur die wirklichen Transporteinnahmen und die wirklichen gesammten Betriebsausgaben zu Grunde gelegt werden. Denn wenn eine Bahn z. B. grosse Werkstätten, Hüttenwerke, Domänen besitzt, aus denen ihr betrüchtliche Einnahmen zufliessen, wie die österr. StaatseisenbahnGesellschaft im Banat, wenn sie grosse disponible Fonds besitzt, aus denen sie erhebliche Zinsen zieht, oder wenn sie an andern Bahnen betheiligt ist wie die S.C.B. an der Bötzbergbahn und der Basler Verbindungsbahu, wenn sie endlich ganze Strecken verpachtet hat und lafür bedeutende Pachtzinse bezieht, oder wenn ihr für Mitbenützung von Bahnhöfen und Bahnstrecken grosse Miethzinse bezahlt werden, wie dies speziell bei vielen schweiz. Bahnen der Fall ist, so dürfen alle diese Eiunahmen nicht als dirckte Betriebseinnalimen gebucht und der Berechnung des Prozentsatzes zu Grunde gelegt werden, weil um diese Einnalımen zu erzielen, der Betrieb keine Ausgaben von Belang zu machen hatte.

Auf der andern Seite gehören allerdings dann auch die bezahlten Miethzinse für fremde und nitbenützte Bahnhöfe und Strecken, sowie 
die Ausgaben des Werkstätten- und Hüttenbetriebes nicht in die Betriebsnusgaben.

Bei der S.C.B. z. B. baben im Jahre 1877 die wirklichen gesammten Betriebsausgaben ca. 53\% der Transporteinnahmen betragen; rechnet naan aber Gesammtausgaben und Gesammteiunalımen gegen einander, so beträgt das Verhältniss nur ca. $47 \%$.

Ein weiterer Faktor, dessen Behandlung auf das Verhältniss der Eimnahmen zu den Ausgaben sehr stark einwirkt, ist sodaun der Erneuerungsfonds, indem dessen Soll und Haben so verschiedenartig gebucht werden kann, dass der vorerwïhnte Prozentsatz bei effektiv gleichen Verhältnissen das eine Mal hoch, das andere Mal nieder herauskommen wird.

Ije Frneuerungsfonds, welche meistens nur bei Privatbahnen bcstehien, sind dazu bestimmt, die Ausgaben fur die Erneuerung von Oberbau und Rollmaterial, deren Betrag ihrer Natur nach von einem Jahre zum andern stark wechseln kann und oft auch stark wechsolt, auf eine lüngere Reihe von Jahren mit möglichster Regelmissigkeit zu vertheilen. Es ist hier nicht der Ort, auf die verschicdenen Grundsätze cinzutreten, welche bei der Einrichtung dieser Fonds befolint zu werden pHegen; der leitende Grundsatz ist der, dass dem Fonds jülırlich eine bestimmte Summe, die bei einzelnen Bahnen mit der Intensitït des Verkehrs variirt, überwiesen wird, und dass dagegen der Fonds für die Kosten von neuen Schienen und Schwellen etc., von neuen Maschinen und Wagen sowie von gewissen Theilen derselben, aufzukommen hat. Nun werden in den ersten Jahren des Betriebes einer Bahn die Ausgaben für diese Zwecke nur klein sein, der Fonds wird also mehr einnehınen als ausgeben, später müssen damn bedeutende Erueuerungen auf einmal vorgenommen werden, der Fonds wird mehr zu leisten haben als seine Zuflüsse betragen und daher abvelumen. Fin ungeführes Gleichbleiben des Fonds, wobei also die jeweilige Einlage immer auch im laufenden Jahre aufgezehrt wiirde, kommt in der Praxis desshalb nicht vor, weil ein Bahnnetz uie lang genug in seinem Bestande unverïndert bleibt, um dieso Ausgleichung zu gestatten, sondern durch Neubauten und Erweiterungen sich stets vergrössert, wesshalb auch die Einnahmen wie die Ausgaben der Fonds fortwährend sich verändern, ganz abgesehen davon, dass die Variationen in den Preisen von Schienen etc. auf die Höhe der Einlage wie der Entnahme ebenfalls einwirken.

Es ist nun bei einzelneu Verwaltungen Sitte, dic Ausgaben für Erneuerungen, welche aus diesem Fonds bestritten werden, nicht in 
die Betriebsrechnung cinzustcllen, während bei andern alle solchen Ausgaben, finden sie nun ihre Deckung wo sie wollen, als Betriebsausgaben gebucht werden. Im erstern Falle werden also die Ausgaben auf don Papicr einen kleinern l3etrag erreichen, und auch der Prozentsatz von Ausgaben zu Einnahmen wird ein günstigerer sein als im zweiten. Bei der Schw. Nordustbahı, welche bis vor einem Jahıre nach der ersten Methode verfuhr, wäre für 1876 ein un ungefïhr $10 \%$ höherer Prozentsatz der Ausgaben herausgekommen als der effektiv angegebene, wenn nach der zweiten Methode gerechnet worden wïre, und also alle Ausgaben für Erneuerung etc. ihren Platz unter den Betriebsausgaben gefunden hätten.

Wenn wir nun in Vorstehenden gesehen, dass uber den Begriff "Betricbsausgaben" ibcrhaupt keine Uebereinstimmung herrscht, so tritt dieser Mangel in erhöltten Maasse au den Tagr, wenn es sich darum handelt, die Ausgaben für bestimmte Dienstzweige auf verschiedenen Bahnen mit einander zu vergleichen. Es wird z. B. viclfach behauptet, dass die "Allgeincine Verwaltung" bei grossen Bahuen relativ weniger koste als bei kleinen, bei Staatsbahnen als bei Akticnbahnen oder auch umgekehrt, und man beruft sich dabei auf die Ziffern der Statistik. Nun wird aber nicht iberall unter "Allgemeiner Verwaltung" das Nümliche verstanden, so dass die betreffenden Ausgabeziffern, wenn sie auch für jede einzclne Bahn absolut richtig sind, sich dennoch zur Vergleichung unter einander in keiner Weise eiguen. Sodann wird in einzelıeu Ländern, wie in Frankreich und der Schweiz, unterschieden zwischen den Kosten des Expeditionsdienstes, d. h. des Stations- und Zugsdienstes einerseits, und denjenigen des Fahrdienstes, d. h. der Maschinen anderseits, während in Deutschland diese beiden Kategorieen von Ausgaben unter "Transportverwaltungs" in einer Summe gegeben werden.

Irgend welche Schlussfolgerungen mit Bezug auf die Ausgaben, welche aus der Vergleichung der Zahlen der verschiedenen Statistiken allein gezogen werden, sind daher immer mit äusscrster Vorsicht aufzunchmen, da sio schr oft durch genauc Vergleichungen - sofern solche überhaupt möglich sind, dementirt werden.

Gehen wir nun zu den Mittheilungen über die Arbeiten der Kommission selbst über, so möge zuerst Einiges über die Zusammensetzung derselben Platz finden.

Auf dem statistisclien Kongress in Budapest wurden nur wenige Mitglieder ernannt, da man sich dort auf die auf jenem Kongress an- 
wesenden Statistiker beschränkte. Da aber die Kommission hauptsïchlich Fachleute enthalten sollte, so wurde dem Bureau derselben, den Herren Brachelli, Hofrath im Handelsministerium und Professor in Wien, Bodio, Direktor der Allgemeinen Statistik und Professor in Rom und Perl, Divisionschef der Grossen Russischen Eiseubahngesellschaft in Petersburg, die Befugniss ertheilt, eine angemessene Allzahl von Eisenbahndirektoren und Beamten der verschicdencu europäischen Staaten zu cooptiren. Ueberdies wurden bei der ersten Session der Kommission, welche im Oktober 1877 in Rom stattfand, wie bei der folgenden in Bern, die Regierungen und Bahnen des betreffenden Landes ersucht, sich durch Delegirte vertreten zu lassen, welche gleiche Rechte wie die ursprünglichen und wie die cooptirten Nitglieder haben, und die auch für die Zukunft als "membres aggrégés" der liommission angehören. Da der Ort der Sitzung jedes Jahr bis zur Vollendung der Aufgabe - ein anderer sein soll, so wird nachgerade die Zahl der Mitglieder aller Art eine ziemlich beträchtliche werden, gegenwärtig beträgt sie 62. In Bern waren anwesend 27 Mitglieder, wovon 6 Schweizer. 'Ausser der Schweiz waren vertreten Russland, Oesterreich, Deutschland, Italien, Holland, Belgien, theilweise ziemlich zahlreich, Frankreich durch zwei Delegirte der Regierung, während die grossen Bahngesellschaften keine $\mathbf{A b g e o r d -}$ ncten gesandt hatten, obwohl sie eingeladen waren, England endlich gar nicht, obwohl Vertreter des Board of trade und solche der grossen Bahnen s. Z. in die Kommission cooptirt worden waren.

Schon in Rom war man ubereingekommen, sich bezliglich der von den Bahnen zu verlangenden Angaben auf das Nothwendigste zu beschränken und für alle Details auf die nationalen Statistiken zu verweisen.

Inımerhin enthält das Formular ca. 260 Kolonnen, während allerdings das neue Schweizerische deren 415 , die deutsche Vereinsstatistik deren 376 (darunter 'aber eine grosse Anzahl mit 2, 3 und 4 Unterrubriken, welche also zwei- und dreifach zu rechnen sind) zählt. Von jenen 260 Kolonnen fallen übrigens auf die Statistik der Unfälle und die der Unterstützungskassen ca. 100, so dass für die eigentliche Bau- und Betriebsstatistik ca. 160 bleiben; eine Zahl, unter welche kaum herabgegangen werden kann, wenn anders alle wirklich interessanten und für die Beurtheilung wichtigen Daten sollen geliefert werden. 
Die 160 Kolonnen sind in 5 Tabellen eingetheilt:

I. Bauverhältnisse.

II. Stand und Leistungen der Betriebsunittel.

III. Anlagekapital.

IV. Einnahmen und Ausgaben.

V. Verwendung des Reinertrags.

Es würde zu weit fuhren, in das Detail dieser 5 Tabellen einzutreten, und es wird genügen, in grossen Zügen zu zeigen, welche Angaben man in denselben finden wird, welche dagegen darin nicht enthalten sind, die doch darin sein sollten, und welche endlich ohne Schaden weggelassen werden könnten.

\section{Bauverhältnisse.}

Aus dieser Tabelle ist ersichtlich die Länge der Bahn, die Spurweite (welche bekanntlich in Russland, Irland und theilweise auch in England, Schweden und Norwegen von der allgemein geltenden von M. 1,435 im Lichten abweicht), das Verhältniss der zwei- und mehrspurigen Strecken zur Gesammtlänge, die Konstruktion des Oberbaues, ob eiserne oder Stahlschienen, ob hölzerne, steinerne oder eiserne Unterlageu. Ferner die Steigungs- und Krümmungsverhältnisse, nümlich die Längen der horizontalen Strecken, der Steigungen bis zu $5 \%$, derjenigen zwischen 5 und $15 \%$ und endlich derjenigen uber $15 \%$. Bei diesem Punkte hätten die schweizerischen Abgeordneten eine Aenderung gewünscht in dem Sinne, dass auch noch die Steigungen von $10 \%$ und von $20 \%$ separat angegeben worden wären, weil auf den schweizerischen Bahnen, wie uberhaupt auf Bahnen in etwas unebenem 'Terrain die Steigung von $5 \%$ nicht mehr die Grenze zwischen leicht zu betreibender Bahn und schwer zu betreibender Bahn darstellt, während allerdings die grossen Hauptbahnen in Frankreich, Deutschland, Oesterreich und England nur ungern über jene $5 \%$ o hinausgegangen sind. Ob der von der Schweiz gestellte bezügliche Antrag Berücksichtigung finden wird, wird erst auf einer nächsten Sitzung entschieden werden, da diese Frage in Bern nicht mehr behandelt werden konnte.

\section{Stand und Leistungender Betriebsmittel.}

Hier wird angegeben die Ausrüstung der Bahnen mit Lokomotiven, Personen- und Güterwagen, absolut und auf den Kilometer Bahnlänge reduzirt, bei den Wagen - um der verschiedenen Bauart derselben Rechnung zu tragen - auch die Zahl der Sitzplätze und 
der Tommen Tragkraft per liilometer. Diese Zahlen sind, wic schon frïher erwälnt, eines der wesentlichsten Momente für die Bedeutung einer Bahn und variiren demgemäss auch ganz enorm. Sie gehen z. B. von 2 Lokomotiven und 8 Personenwagen per Kilometer auf der unterirdischen Eisenbahn in London (Metropolitan Railway) herunter bis auf 0,1 und 0,07 Lokomotiven und 0,3 und 0,4 Personenwagen per Kilometer auf der Dniester-, der Donau-Drau- und der Vorarlbergerbahu; dafür sind allerdings auf der Metropolitan (ca. $22 \mathrm{Kil}$. lang) im Jahr 1876 über 50 Millionen Personen befördert worden, auf der Dniesterbahn dagegen (113 Kilometer lang) nur 156,000 Personen.

Sodann wird angegeben die durchsclunittlich per Lokomotive, per Personen- und per Güterwagen zurückgelegte Distanz; es gibt dics in gewisscm Sinue eineu Maassstab für die Güte der Verwaltung, indeu jede Verwaltung trachten muss, ihren Verkehr mit möglichst wenig Material zu bewältigen. Fine Normalziffer aufzustellen, geht aber auch hier nicht an, da bei starkem Verkehr die Leistungen des Materials in der Regel auch stärker sein werden als bei schwachem, und weil besonders ein sehr schwankender Verkehr - für Personen ein staker Sonntags- oder Fremdeuverkehr, für Güter z. B. der Getreideund Kohlenverkehr, der mit den Jahreszciten stark variirt, - einen weit grössern Vorrath von Rollmaterial erfordert, als cin ebenso grosser Gesammtverkehr, der regelmässig auf das ganze Jahr vertheilt ist. In dritter Linie gibt die Tabelle II die hauptsächlichsten Angaben iiber die Transportquantitäten - Personen und Güter, sowohl die absolute Auzahl Personen und Tonnen, welcho überhaupt befürdert worden sind, als auch den von ihnen zurückgelegten Weg - die Personen- und die Tonnenkilometer - und endlich das Verhältniss, in welchem die transportirten Sitzplätze und der transportirte Laderaum ausgenützt worden ist. Die absolute Zahl von Personen und Tonnen hat nur ein sehr beschuänktes Interesse, da in dieser Zahl eine Person, die von Basel nach Muttenz geht, ebenso viel zïhlt als eiue von Bodenbach nach Orsowa oder von Paris nach Mentone reiscude, während diese so verschicdenen linheiten eigentlich nicht vergleichbar sind. Diese Angaben sind denn auch vermuthlich nur aufgenommeu worden, weil man voraussah, dass die weitergehenden Zahlen von einzelnen Staaten, speziell von England, nicht würden geliefert werden.

Ein richtiges Bild des Verkehrs einer Bahn kann nämlich nur eine Zahl oder eine Reihe von Zahlen geben, welche auch die Entfernungen, auf welche Personen und Güter transportirt worden sind, 
zun Ausdruck bringt, also die Anzahl Personenkilometer, die auf der ganzen $B$ ahn zuruckgelegt worden sind, und am besten die Anzahl Personen, welche uiber die ganze Bahn transportirt worden sind, gleich dem Quotient aus der Gesammtzahl Personenkilometer durch die Lïnge der Bahn. Z. B. die Bahn ist 20 Kilom. Jang: 9 Personen fahren je 5 Kil. weit, 5 Personen 10 Kil. und 7 Personen 15 Kil. weit, so laben dic 21 Personen zurückgelegt $45+50+105=200$ Personenkilometer, oder gleichviel, als wenn 10 Personen uber die ganze Bahn gefahren wären. Diese Zahl 10 wird auch der spezifische Personenverkelır der Bahn genannt. Ganz auf dieselbe Weise wird der spezifische Giiterverkehr berechnet.

Es eignen sich die Zahlen des spezifischen Verkehrs desshalb sehr gut \%u Vergleichungen, weil die Länge der Bahn nicht weiter berücksichtigt zu werden braucht. Werden nur die absoluten Zahlen von Personen und Tonmen auf verschiedeneu Balınen einander gegenübergestellt, so erhalten sie - ganz abgesehen davon, dass der zurïckgrelegte Weg dabei keinen Ausdruck findet, - einige Bedeutung erst, wenn die Läuge der betr. Bahnen mit in Betracht gezogen wird, weil eine längere Bahn in der Regel an und für sich mehr Reisende Laben wird als eine kürzere.

In der Zahl des spezifischen Verkehrs ist dagegen Alles vereinigt: der Einfluss der absoluten Zahl der Reisenden oder Tonnen, des von denselben zurückgelegten Weges und der Länge der Bahn, und die Einheit ist dieselbe bei der S.C.B. wie z. B. bei der französischen Ostbahn oder der Cöln-Mindener-Bahn.

Der spezifische Verkehr beträgt auf diesen Bahnen:

$\begin{array}{lcrrrc}\text { S.C.B. } & 1877 & 263,713 & \text { Pers. } & 177,541 & \text { Tonuen } \\ \text { Est français a. r. } & 1873 & 455,586 & " & 888,109 & " \\ \text { Paris d Vincennes } & \text { " } & 2,129,357 & " & - & " \\ \text { Cöln-Minden } & " & 374,349 & \text { " } & 1,014,112 & "\end{array}$

Die Berechnung dieser Zahlen ist eine etwas zeitraubende Arbeit, da für jeden lleisenden und jede Tonne der zurückgelegte Weg bekaunt sein muss, was eine Menge von Aufschreibungen voraussetzt, welche sich die Englünder z. B. ersparen. Es ist auch nicht zu erwarten, dass sie es der internationalen Eisenbahnstatistik zu Liebe anfangen werden zu thun, und so werden voraussichtlich immer empfindliche Lücken in den Angaben derselben bestehen bleiben.

Die Gesammtzahl der Personen- und T'onnenkilometer ist nämlich ausserdem noch die Basis für die Berechnung der Ausnützung des Materials. Werden die im Laufe des Jahres zurückgelegten Sitzplatz- 
kilometer durch die Personenkilometer dividirt, so ergibt sich dio Anzahl Plätze, welche auf einen Reisenden transportirt worden sind oder umgekehrt der Prozentsatz der besetzten Plätze zu den überhaupt angebotenen und trausportirten. Dieser Prozentsatz ist immer sehr gering, in der Schweiz des grossen Lokalverkehres wegen relativ gilnstig - ca. $33 \%$, in Deutschland dagegen nur ca. $25 \%$ - mit andern Worten, in der Schweiz ist von 3 Plätzen nur eincr besetzt, in Deutschland von 4 Plätzen nur einer. Natürlich ist hier der Durchschnitt des ganzen Jahres gemeint, denn in der Reisesaison und bei den bequemen Zügen sowie auf den frequenteren Strecken müssen die Plätze weit besser besetzt sein, um die oft äusserst geringe Frequenz im Winter oder bei Frulhzügen etc. zu kompensiren. In den cinzeluen Klassen ist das Verhältniss ebenfalls verschieden, in der ersten Klasse ist nur ungefähr $1 / 7$ aller Plätze besetzt, in der III. Kl. eber mehr als $1 / 3$. Beiläufig bemerkt, beweist der Prozentsatz der I. Kl., dass die Taxen derselben viel zu nieder sind, und es gibt sich auch das allgemeine Bestreben kund, die Verwendung derselben möglichst einzuschränken, da auf dersclben gegenwärtig nichts verdient wird. Auf einer schweizerischen Nebeubahn, wo dieselbe seit zwei Jahren allerdings abgeschafft ist, war im Jahre 1874 von ca. 700 transportirten Plătzen I. Kl. nur einer besetzt, und die Einnahme reichte ungefähr hin, um einen Drittel der Unterhaltungskosten der Wagen I. Kl. zu bestreiten.

Beim Gutterverkehr wird die Ausnützung der Tragfähigkeit auf die nämliche Art wie beim Personenverkehr berechnet. Das Verhältniss ist in der Schweiz ca. 27-30\%, in Deutschland ca. 40\%. Es kann auch in der Schweiz nicht so gut sein als in Deutschland, weil der Massenverkehr (Kohle, Getreide etc.) in der Schweiz nur in einer Richtung stattfindet, eine Rückfracht also fur die Kohlen- etc. Wagen nicht vorhanden ist. (Ueber den Hauenstein auf der Linie BaselOlten gingen im Jahre 1874 6mal mehr Guter von Nord nach Sud als von Süd nach Nord.) Dazu kommt noch, dass eine Menge Guter so beschaffen sind, dass die Wagen gar nicht bis zur vollen Tragkraft damit beladen werden können (Wein, Baumwolle, Wolle, Rinde, Heu, Stroh, Torf etc. etc.), so dass eine erhebliche Verbesserung des Prozentsatzes von $27-30 \%$ in der Schweiz nicht zu erwarten ist. Für England sind diese Zahlen nicht bekannt, weil, wio schon erwälınt, die Grundlagen dazu, die Gesammtzahl der Tonnenkilometer, nicht erhoben wird. Nach dem, was sonst uber diesen Punkt bekannt ist, würde der Prozentsatź für England schr nieder 
ausfallen, weil dort - für die General Merchandise wenigstens hauptsächlich die Schnelligkeit der Beförderung ins Gewicht fällt, und die Bahnen, welche im Allgemeinen hohe Taxen beziehen können, vorziehen, einen Wagen zu nur $15 \%$ beladen abgeben zu lassen, elıer als zu warten, ob in Lauf des Tages weitere Güter nach derselben Bestimmung dazu kommen, welche die Tragfähigkeit des Wagens besser auszunuitzen gestatten würden. Eilgut ist in England so gut als unbekannt, weil schon das gewöhnliche Gut - immer mit Ausnahme der sog. Minerals: Kohle, Erze etc. - mit Eilgutgeschwindigkeit befördert wird. Der Kaufmann in Manchester weiss gar nichts Anderes, als dass eine Waare, die heute in London der Bahn übergeben wird, morgen frub in seinem Magazin in Manchester liegt.

\section{A nlagekapital.}

Das Aulagekapital soll angegeben werden nach den einzelnen Katerorieen -- Aktien, Subventionen, Aulehen, Obligationen (eine deutliche Defiuition dicser letztern Ausdrucke ist nicht gegeben) und zwar nach dem Nominalbetrag der Titel. Dicse letztere Bestimmung wird voraussichtlich gestrichen werden müssen, wenn anders die Zahlen der Statistik wirklichen Werth haben sollen.

Wurde derselben strikte nachgekommen, so würde das Anlagekapital der französischen Bahnen um ca. die Hälfte höher erscheinen als es wirklich ist. In Frankreich ist nämlich weitaus der grösste Theil des Anlagekapitals der Bahnen - bei der Paris-Lyon-Méditerranée-Balın 2. B. Aktien

Fr. 350 Mill.,

Obligationen ancien réseau 1723

$\left.\begin{array}{ll}\text { nouveau " } & 555 \\ \text { Algérie } & 144 \\ \text { Mont Cenis } & 107\end{array}\right\}$ " 2529

vermittelst $3 \%$ iger Obligationen von nom. Fr. 500 mit theilweiser Staatsgarantie aufgebracht worden, welche je nach dem Stande des Geldmarktes zu Fr. 280 bis lir. 340 ausgegeben worden sind. In deu Bilauzen der frauzösischen Bahnen sind diese Obligationen nur mit dem Betrage aufgeführt, den sie der Gesellschaft wirklich eingebracht haben, z. B. Nord ancien Réseau Dec. 1874

1,162,855 Oblig. $3 \%$ nominell von Fr. 500 Fr. 346,065,244 $=$ Fr. 297 p. Stück.

Wenn diese Obligationen zu ihrem Nominalwerth von F. 500 per Stiuck gerechnet werden miussten, so würden die Erstellungskosten in 
ganz ungerechtfertigter Weise in die Höhe getrieben werden und eine richtige Vergleichung mit anderen Ländern gar vicht melır gestatten.

\section{Einnahmen und Ausgaben.}

Ueber die Klassifizirung der Einnahmen herrscht im Allgemeinen Uebereinstimmung, mit Ausnahme des Postens "Gepäckeiunahmen", welche die Einen unter Personenverkehr rubriziren, weil olne Persouenverkehr keine Gepäckeinnahmen denkbar sind, während die Andern sie dem Güterverkehr zutheilen, weil der Parcours der Gepäckwagen mit dem der Güterwagen zusammengezählt wird. Die Frage ist nicht sehr wichtig und wurde daher auch nicht weiter erörtert. -

Ueber die Rubrizirung der Ausgaben waren dagegen die Meinungen sehr getheilt, und zwar sowohl snit Bezug auf die Eintheiluug derselben in die 4 Rubriken:

a) Allgemeine Verwaltung,

b) Bahnaufsicht und -Unterhalt,

c) Expeditions- und Zugsdienst,

d) Fahrdienst (Traction),

als auch mit Bezug auf die Berechnung der Ausgaben für Erneuerung von Oberbau- und Rollmaterial, welche, wie schon erwiihnt, sehr verschieden aufgefasst wird.

Nach langen Verhandlungen und zum 'Theil etwas verworrenen Debatten gelangte man zu einer prinzipiellen Einigung, welche, wenn auch die angenommene Redaktion vielleicht nicht allseitig befriedigt, doch bei nochmaliger Behandlung des Gegenstandes die Erzielung einer definitiven Uebereinstimmung hoffen lässt. Die Standpunkte waren so entgegengesetzt, und die Verbältuisse der Bahnen mit Erneuerungsfonds den Vertretern der Bahnen ohne Erneuerungsfonds theilweise wenig geläufig, überdies die Diskussion wegen der verschiedenen Sprachen nicht immer ganz allgemein verständlich, dass eine definitive Erledigung der streitigen Punkte kaum zu erwarten war, und so einigte man sich schliesslich nur im Prinzip dahin, dass alle Ausgaben für Erneuerung, ob sie nun direkt aus dem Betrieb oder aus dem Erneuerungsfonds bestritten werden, in die Betriebsausgaben eingesetzt werden sollen, immerhin mit Unterscheidung der Art der Bestreitung.

Leider gehörte es nicht in das Programm der Berner Konferenz, auch uber die allgemeine Rubrizirung der Einnalımen und der Ausgaben eine Meinung abzugeben, und so kam die Frage der Berech- 
nung des Prozenitsatzes von Einnahmen zu Ausgaben gar nicht zur Diskussion. Das internationale Formular ist in dieser Beziehung ungenügend, indem die indirekten Einnahmen und Ausgaben von den wirklichen Transporteinmahmen und Betriebsausgaben gar nicht oder nicht deutlich genug ausgeschieden sind. Es wird desshalb auch in dieser Bezielıung einer spätern Versammlung vorbehalten bleiben, Abhülfe zu treffen.

Fs ist nun allerdings zuzugcben, dass . für die grossen Bahnnetze des Auslandes und besonders für die Durchschnittszahlen gauzer Lünder, diese indirekten Einuahmen und Ausgaben viel weniger ins Gewicht fallen als in unsern schweizerischen Verhältuissen, wo die Mitbeuützung von Bahnhöfen und Bahustrecken selır ausgebildet ist, und bei der relativen Kleinheit der einzelnen Netze für die Rechnungsstellung sehr ins Gewicht fältt, und wo bei einzelnen Bahnen die indirekten Einnahmen (im Sinue von pag. 97) bis zu 20 und $25 \%$ der Totaleinnahmen ausmachen.

Weil aber in der internationalen Statistik nicht nur die Durchschuittsresultate ganzer Länder gegeben werden sollen, sondern ebensowohl die Resultate jeder einzelnen bahn, so ist es für die schweizerischen Bahnen von Bedeutung, dass die Rubrizirung von Einnahmen und Ausgaben auf eine Weise stattfinde, dass auch ihre Verhältnisse zu einem klaren und richtigen Ausdrucke kommen.

Die schweizerischen Vertreter haben desshalb in einer Spezialeingabe auf diesen Punkt mit allem Nachdruck hingewieselı, und es ist zu erwarteu, dass ihren Vorstellungen Rechnung getragen werde.

\section{Verwendung des Betriebsuberschusses.}

Der oben hervorgehobene Mangel des internationalen Formulars, betr. die Einnahmen und Ausgaben, hat zur Folge, dass die Angaben ubcr die Verwendung des Betriebsüberschusses über einzelne wichtige Punkte keinen Aufschluss ertheilen und desshalb noch revidirt werden sollten.

Streng genommen stellt der „Betriebsüberschuss“ nur das finanzielle Resultat des eigentlichen Bahnbetriebes dar, ohne Berücksichtigung allfälliger anderer Einnalımen der Unternehınung (vide pag. 97), also den Ueberscliuss der Transporteinnahmen über die eigeutlichen Betriebsausgaben; dieser Betrag kann dann per Bahnkilometer, per Zugsoder Achskilometer berechnet werden und gielt ein Bild der Resultate des Bahubetriebs an sich; der "Reinertrag" dagegen den Ueber- 
schuss der Gesammteinnahmen über alle Ausgaben: direkte und indirekte, Zinsen der Anlehen, Amortisation derselben, Einlage in die Erneuerungsfonds etc.; m. a. W. denjenigen Betrag, welcher zur Remuneration des eigentlichen Gesellschaftskapitals, der Aktien, disponibel ist, und aus dieser Summe kann die Verzinsung des in der Unteruehmung engagirten Stammkapitals berechnet werden. Dieser Betrag braucht allerdings nicht ausschliesslich zur Vertheilung an die Aktionäre verwendet zu werden, sondern es können auch gewisse Betrïge in einen Reservefonds fallen, andere $\mathrm{zu}$ Abschreibungen auf Materialien oder ähnlich dienen. Der Grundsatz wäre also der, dass alle Zahlungen, welche auf eingegangenen Verpflichtungen beruhen, von den Gesamint-Einnahmen abgezogen werden mussen, ehe und bevor von Reinertrag geredet werden kann.

Eine solche klar definirte Summe ist nun aus dem internationalen Formular nicht zu entnehmen; so wird z. B. der Agioverlust, der bei einzelnen österreichischen Bahnen bedeutende Summen repräsentirt, erst nach den Aktiendividenden aufgeführt, während doch diese letztern erst ausbezahlt werden können, wenn der Agioverlust auf den Obligationszinsen gedeckt ist.

Das Vorstehende wäre ungefähr der Inhalt der 5 Tableaux und der 160 Kolounen. Wie sie aus den dazu gemachten Bemerkungen haben entuebmen können, ist nicht Alles als vollständig zweckentspreclsend zu betrachten, und es wird noch mehr als eine Zusammenkunft der Kommission erfordern, um das Formular endgültig festzustellen.

Der in Rom 1877 vereinbarte Entwurf wurde im Laufe 1878 allen europäischen Eisenbahnverwaltungen zur Ausfullung und Begutachtung mitgetheilt, und es waren auch bis zur Berner Session eine Reihe von Eingaben mit Abänderungsanträgen, von denen z. B. diejenige des Vercins deutscher Eisenbahnverwaltungen nicht weniger als 16 Folioseiten füllt, eingegangen. Alle diese Anträge sollen in der năchsten Zusammenkunft behandelt werden.

Es ist hier vielleicht der Ort, uber den Gang der Verhandlungen einige Worte zu sagen. In Bern, wie schon in Rom, wurde gleich bei Beginn der Verhandlungen von verschiedenen Seiten vorgeschlagen, für die einzelnen Tabellen Kommissionen einzusetzen, die die Fragen in Detail zu prüfen hätten und nur die grundsätzlichen Differenzen dem Plenum zur Entscheidung vorlegen würden. Diese Vorschläge, durcl welche eine kostbare Zeit erspart worden wäre, wurden durch 
einzelne Staaten, hauptsächlich Belgien, Frankreich und Italien mit grossem Nachdruck bekämpft und in Folge dessen bei der Abstimmung nicht angenonmen, so dass die Diskussion im Plenum stattfand, wobei besonders wegen der Verschiedenheit der Sprachen die Substanz der oft sehr einlässlichen Voten vielfach nicht richtig aufgefasst wurde. Es ist aber nur billig, zu konstatiren, dass die Leitung der Diskussion wegen der oft sehr ins Spezielle grchenden Erörterungen, denen nur ganz Eingeweihte folgen konnten, und wegen der ganz von einander abweichenden Standpunkte der Sprechenden sehr schwierig und ermiidend sein musste, ein Uebelstand, der indessen bei Verlegung der Detaildebatten in die Spezialkounmissionen in holem Grade veruindert worden wäre.

Ausser den bis jetzt besprochenen 5 Tabellen wurden in Bern noch bchaudelt

die Frage der Waarenstatistik,

die Unfallstatistik, und

die Statistik der Hülfs-, Pensions- und Krankenkassen bei Eisenbahnen.

Ich erlaube mir, die beiden letztern Punkte ganz unberührt zu lassen, da eine auch nur oberflüchliche Berichterstattung darüber zu weit führen würde.

Betreffend die erste Frage, die Statistik der Waarenbewegung auf den Eisenbahnen lag ein Referat des russischen Vicepräsidenten Herrn Perl vor, das dahin ging, es sollten alle Eisenbahnen die Bewegung der hauptsächlichsten auf ihrem Netze zur Beförderung gelangenden Waaren feststellen und in graphischen Tableaux zur Anschauung bringen. Im Anschluss an diesen Antrag legte der andere russische Vertreter, Herr $\mathrm{Bloch}$, einen Atlas vor, enthaltend die graphisclue Darstelluug der Bewegung von 12 Hauptartikeln - Getreide, Holz, Hanf, 'Talg, Kohle, Eisen etc. - auf allen russischen Bahnen. Nach einer längern Diskussion, in welcher das Verdieustliche der Anregung selbst wie auch der bereits unternommenen Arbeiten vollständig anerkannt wurde, wurde beschlossen, für diese Frage eine Subkommission zu bestellen, welche im Mai 1879 in Heidelberg zusammentreten und der grossen Kommission bezügl. Detailanträge bringen sollte.

Es machten sich in der Diskussion mannigfache Bedenken geltend über die Möglichkeit, eine solche Waarenstatistik aufzustellen, über die Widersprliche, welche zwischen dieser Eisenbahn-Waarenstatistik und der allgemeinen Zollstatistik der einzelnen Länder nothwendig XXXIY. 
bestehen müssten, über die Unzuverlässigkeit der Schlussfolgerungen, die aus den Zahlen derselben gezogen würden, sowie nicht zum Mindesten über die Bereitwilligkeit der Bahnen, die erforderlichen Daten zu liefern, während über die Wünschbarkeit einer andern als der bisherigen Behandlung dor Waarenbewegung auf den Eisenbahnen nur eine Stinme herrschte.

Auch die Darstellung der Resultate auf graphischem Wege fand allgemeine Zustimmung.

Aus meinen Erörterungen werden Sie vielleicht den Eindruck erhalten haben, dass die Eisenbahnstatistik überhaupt noch sehr unvollkommen sein muss, da ich Ihnen melir von den Mängeln als von deu guten Eigenschaften derselben gesprochen habe, und dass auch dic Arbeiten der internationalen Kommission keinen sehr grossen Werth haben.

Ich wöchte aber doch nicht, dass den also wäre. Es wäre ungerecht, das viele Gute der bestehenden Statistiken zu verkennen, und der Schluss, den ich aus den Differenzen, die zwischen den einzelnen derselben bestehen und aus der theilweisen Mangelhaftigkeit der Arbeiten ziehe, ist nur der, dass es nicht angeht, einzelne Zahlen herauszugreifen und, darauf gestiutzt, Systeme zu bauen oder gewisse Ansichten als unumstösslich bewiesen darzustellen, sondern dass vielmelır beim Gebrauch dieser Zahlen äusserste Vorsicht nothwendig ist, und jeweilen alle Verhältnisse sorgfältig mit in Betracht gezogen werden müssen.

Und was die Arbeiten der internationalen Kommission betrifft, so sind sie ja gewiss nicht vollkommen, und werden vielleicht noch längere Zeit nicht zu dem Ziele führen, das sie jetzt im Auge haben. Denn abgesehen davon, dass eine prinzipielle Einigung uber die verschiedenen Differeuzpunkte im Schoosse der Kommission nicht so bald wird erreicht werden, so bleibt immer noch - auch wenn diese Schwierigkcit gelöst sein wird - die grosse Frage zu beantworten, mit welchen Mitteln die einzelnen Verwaltungen angehalten werden können, die Angaben zu liefern. Bis jetzt ist die Kommission eine reine Privatsache, und es wird sich demnach darum handeln, wenn ein endgültiges Formular festgestellt sein wird, dasselbe auf dem Wege von offiziellen Konferenzen durch die einzelnen Staaten genehmigen zu lassen, damit die Ausfüllung durch die oftiziellen Eiseubahnämter oder Ministerien vorgenommen wird. 
Weln aber auch dieses Ziel in den nächsten Jahren noch nicht erreicht werden sollte, so werden meines Erachtens die Zusammenkulufte der Kommission nicht fruchtlos bleiben, die Einigung wird sich, wenn nicht vollständig und in kurzer Zeit, doch wenigstens in einigen Hauptpunkten nach Verfluss einer kürzern oder längern Reihe von Jahren vollziehen, und es verdienen daher die Arbeiten der internatioualen Kommission und besonders ihres Bureau's unsere vollste Anerkennung. 